$\mathrm{MAD} / \mathrm{TH}-92-04$

hep-th/9302137

December 1992

\title{
Harmonic BRST Quantization of Systems with Irreducible Holomorphic Boson and Fermion Constraints
}

\author{
Theodore J. Allen and Dennis B. Crossley \\ Department of Physics \\ 1150 University Avenue \\ University of Wisconsin, Madison, WI 53706 USA
}

\begin{abstract}
We show that the harmonic Becchi-Rouet-Stora-Tyutin method of quantizing bosonic systems with second-class constraints or first-class holomorphic constraints extends to systems having both bosonic and fermionic second-class or first-class holomorphic constraints. Using a limit argument, we show that the harmonic BRST modified path integral reproduces the correct Senjanovic measure.
\end{abstract}




\section{Introduction}

A new implementation of Becchi-Rouet-Stora-Tyutin quantization was introduced recently by one of the authors in order to quantize theories with bosonic holomorphic constraints. ${ }^{[1]}$ The main assumption of the method is that there is an algebra of first-class constraints, some of which are not real-valued, but rather are holomorphic, functions and that some subset of the holomorphic constraints, together with their complex conjugates, are second-class. That is, the matrix of Poisson brackets of the holomorphic with their anti-holomorphic partners is not weakly vanishing. If the matrix of Poisson brackets were weakly vanishing, one could simply take the real and imaginary parts of the constraints as being separate first-class constraints and ignore altogether the difficulties of any holomorphic structure. Keeping the holomorphic structure is impossible in a standard Becchi-Rouet-Stora-Tyutin-Batalin-Fradkin-Vilkovisky quantization. The quantum constraints and, hence, the BRST-BFV charge operator will not be hermitian, making it impossible to decouple the unphysical states from the physical ones.

The number of systems to which our method applies is in principle quite large. Kalau ${ }^{[2]}$ has shown that any set of an even number of second-class constraints may be split into holomorphic and anti-holomorphic algebras, but has noted that the split may not be computationally useful, either because the quantum algebra may have anomalies or because the holomorphic constraints are computationally intricate. Perhaps the most important example in which a holomorphic structure is useful is that of Ashtekar's new canonical variables reformulation of general relativity, ${ }^{[3]}$ where all the constraints are first-class holomorphic and polynomial when written in terms of the self-dual spin connection. Other examples are the $D=10$ harmonic superstring and superparticle ${ }^{[4,5]}$ the Brink-Schwarz superparticle in four dimensions ${ }^{[5,6]}$ and certain coadjoint orbit theories such as particle spin dynamics on a Lie group. ${ }^{[7]}$ We remark that this splitting can also be used with the operatorial quantization in the case that classical first-class constraints become anomalous in the quantum theory, but our path-integral may need to be modified along the lines of ref. [8]. Operatorial constructions somewhat different from ours have been given by Hasiewicz et al. ${ }^{[9]}$ and recently by Marnelius. ${ }^{[10]}$ The only example of a system known to us to which our method does not apply in principle is Berezin and Marinov's ${ }^{[11]}$ action for a massive point fermion which has an odd number of second-class fermionic constraints.

The present work extends the harmonic BRST-BFV quantization scheme to the case of both bosonic and fermionic constraints. In section 2 we review the use of the harmonic BRST-BFV method for systems with bosonic constraints. In section 3 we demonstrate the extension of this method to systems with fermionic constraints by applying it to the most trivial case, that of a single fermionic constraint. In section 4 we treat the general case of an arbitrary number of bosonic and fermionic constraints. We demonstrate that 
in a certain limit, the modification of the path integral reproduces the correct Senjanovic ${ }^{[12]}$ measure for second-class constraints. Throughout the analysis we have assumed that the constraints are irreducible.

\section{The Harmonic BRST-BFV Method for Bosonic Constraints}

The main assumption that we start from is the existence of an algebra of holomorphic constraints, $\mathcal{A}$, closed under Poisson brackets, whose matrix of Poisson brackets with the complex conjugate algebra, $\overline{\mathcal{A}}$, has non-vanishing determinant, even weakly,

$$
\operatorname{det}_{(i j)}\left\{a_{i}, \bar{a}_{j}\right\} \not \approx 0, \quad a_{i} \in \mathcal{A}, \quad \bar{a}_{j} \in \overline{\mathcal{A}}
$$

If the determinant in (1) were zero, we could find a subalgebra, $\mathcal{B} \subset \mathcal{A}$, such that the determinant was non-vanishing for $a_{i} \in \mathcal{B}, \bar{a}_{j} \in \overline{\mathcal{B}}$. We could then take the real and imaginary parts of the remaining constraints $a_{i} \in \mathcal{A} \backslash \mathcal{B}$ and treat them separately.

We also assume that there may be some first-class constraints, $\mathcal{F}$, as well, which we may take to be real. To construct the necessary operators, we look at the one-parameter set of algebras, $\mathcal{F} \oplus t \mathcal{A}$, consisting of the first-class constraints and holomorphic constraints scaled by an arbitrary real parameter $t$. It is well known that there exists a fermionic function, the formal BRST charge, which has vanishing Poisson bracket with itself, ${ }^{[13]}$

$$
\begin{aligned}
Q(\mathcal{F} \oplus t \mathcal{A}) & =\eta_{I} f_{I}+t \eta_{i} a_{i}+\ldots \\
& =\Omega+t \Theta \\
\{Q, Q\} & =0
\end{aligned}
$$

In eq. (2), the $\eta$ 's are ghost variables, which are anticommuting when the constraints are bosonic. It is worth pointing out that the charge $\Omega$ is not necessarily identical to the BRST charge used when the second-class constraints are implemented using Dirac brackets. For an example of this, see ref. [1].

Because the parameter $t$ is arbitrary, the last equality of (2) implies the following relations,

$$
\{\Omega, \Omega\}=\{\Omega, \Theta\}=\{\Theta, \Theta\}=0
$$

Unfortunately, the charge $Q$ defined in (2) is not real, so it is not suitable to use its quantum version, $\hat{Q}$, to define physical states. It is necessary for the existence of a BRST cohomology that the operator $\hat{Q}$ 
be either hermitian or anti-hermitian. This is because the equivalence relation $|\Psi\rangle \cong|\Psi\rangle+\hat{Q}|\Phi\rangle$, with $|\Phi\rangle$ arbitrary, must be compatible with the inner product on the Hilbert space. In other words, states that are $\hat{Q}$-exact must be orthogonal to the physical $\hat{Q}$-closed (and $\hat{Q}$-exact) states and, therefore, have zero norm. To have the decoupling

$$
\langle\Psi \mid \hat{Q} \Phi\rangle=\left\langle\hat{Q}^{\dagger} \Psi \mid \Phi\right\rangle=0
$$

for all physical states $|\Psi\rangle$ and all arbitrary $|\Phi\rangle$, it is necessary that $\hat{Q}= \pm \hat{Q}^{\dagger}$.

It is useful to use the operators $\hat{\Omega}$ and $\hat{\Theta}$ separately. Physical states are defined to be those which are in the cohomology of $\hat{\Omega}$ and are annihilated by both $\hat{\Theta}$ and its adjoint, $\hat{\bar{\Theta}}$,

$$
\begin{gathered}
\hat{\Theta} \mid \text { phys }\rangle=\hat{\bar{\Theta}} \mid \text { phys }\rangle=\hat{\Omega} \mid \text { phys }\rangle=0, \\
\mid \text { phys }\rangle \cong \mid \text { phys }\rangle+\hat{\Omega} \mid \text { anything }\rangle .
\end{gathered}
$$

These states are harmonic in the sense that they are annihilated by the Laplacian $\{\hat{\Theta}, \hat{\bar{\Theta}}\}$.

The ghosts used in $\Theta$ and $\bar{\Theta}$ are different from the usual BRST ghosts. There are two inequivalent complex structures one can impose on fermionic phase space variables. The one closest in analogy to the bosonic oscillator has the canonically conjugate variables, $\xi$ and $\xi^{*}=\bar{\xi}$, which are also complex conjugates of one another. The real and imaginary parts of $\xi$, in addition to being real, are also canonically self-conjugate,

$$
\begin{aligned}
& \{\xi, \bar{\xi}\}=-i, \quad \xi=\frac{1}{\sqrt{2}}(\rho+i \pi), \\
& \{\rho, \rho\}=\{\pi, \pi\}=-i, \quad\{\rho, \pi\}=0 .
\end{aligned}
$$

These are the ghosts that we will require. They differ from the $(b, c)$ ghosts $^{[14,15]}$ of the usual BRST-BFV formalism. The $(b, c)$ ghosts are real and are not canonically self-conjugate,

$$
\{b, c\}=-i, \quad\{b, b\}=\{c, c\}=0, \quad b^{*}=b, \quad c^{*}=c .
$$

The symmetry of the fermionic Poisson bracket allows the existence of these two inequivalent complex structures on a pair of canonically conjugate fermionic variables. Bosons have only one such structure. The ghosts used to construct $\Theta$ for the holomorphic constraints $a_{i} \in \mathcal{A}$ are of the first type (6), while the ghosts used in $\Omega$ are the $(b, c)$ ghosts. 
In ref. [1] a Hamiltonian path integral construction was given, analogous to the $\mathrm{BFV}^{[16]}$ construction explained in great detail in ref. [13]. When the assumption of the holomorphic BRST-BFV method applies, the Hamiltonian path integral is

$$
\mathcal{Z}_{\Psi, \beta}=\int \mathcal{D} \mu \exp \frac{i}{\hbar} \int d t\left(i \bar{\xi} \dot{\xi}+p \dot{q}+b \dot{c}+\bar{c} \dot{\bar{b}}+\pi \dot{\lambda}-H_{B R S T}-\beta\{\Theta, \bar{\Theta}\}-\{\Omega, \Psi\}\right)
$$

If the fermionic gauge-fixing parameter $\Psi$ and the constant $\beta$ are both imaginary, then the path integral is manifestly unitary. One can show that the path integral (8) is invariant under infinitesimal variations of $\Psi$ and $\beta$. The "extra" piece, $\exp (-i \beta\{\Theta, \bar{\Theta}\})$, in (8) in the limit of $\beta \rightarrow \infty$ becomes the correct Senjanovic measure for second-class constraints and eliminates the ghost degrees of freedom as well,

$$
\lim _{\beta \rightarrow \infty} e^{-i \beta\{\Theta, \bar{\Theta}\}}=(-1)^{N} \pi^{N} \delta^{N}\left(\xi_{i}\right) \delta^{N}\left(\bar{\xi}_{j}\right) \operatorname{det}\left(i\left\{a_{i}, \bar{a}_{j}\right\}\right) \delta^{N}\left(\operatorname{Re} a_{i}\right) \delta^{N}\left(\operatorname{Im} a_{j}\right)
$$

\section{Fermionic Constraints}

We first consider the simple case of a single holomorphic fermionic constraint, $\phi \approx 0$. Since both the real and imaginary parts of $\phi$ must weakly vanish, it follows that the complex conjugate constraint also vanishes, $\bar{\phi} \approx 0$. The harmonic BRST-BFV method then introduces a pair of bosonic ghosts, which are both complex conjugate and canonically conjugate. A single fermionic constraint can have only a very simple algebra, although one that is more general than that of a single bosonic constraint. That algebra is

$$
\{\phi, \phi\}=\gamma \phi
$$

where $\gamma$ is a fermionic function on phase space. For simplicity, we assume that the brackets between $\phi$ and $\bar{\phi}$ are those of the fermionic oscillator, $\{\phi, \bar{\phi}\}=-i$. We consider explicitly the case in which $\gamma$ is a (Grassmann odd) constant. In this case the method yields a state identical to that of a single bosonic constraint, but with the roles of the original and the ghost variables interchanged. This is a consequence of the $O S p(1,1 \mid 2)$ invariance of the system. ${ }^{[1]}$

The harmonic BRST charges for (1) are

$$
\hat{\Theta}=\hat{c} \hat{\phi}+\frac{i}{2} \hat{\gamma} \hat{\bar{c}} \hat{c} \hat{c}, \quad \hat{\bar{\Theta}}=\hat{\bar{c}} \hat{\bar{\phi}}-\frac{i}{2} \hat{\bar{\gamma}} \hat{\bar{c}} \hat{\bar{c}} \hat{c}
$$

The most general state in the ghost-enlarged Hilbert space is a sum of products of ghost states, $|n\rangle_{c}$, of occupation number $n$ with states, $\left|\psi_{n}\right\rangle$, of the original Hilbert space: $|\Psi\rangle=\sum_{n=0}^{\infty}|n\rangle_{c}\left|\psi_{n}\right\rangle$. The harmonicity 
conditions (5) yield the physical state

$$
\mid \text { phys }\rangle=|0\rangle_{c}\left|\psi_{0}\right\rangle, \quad \hat{\bar{\phi}}\left|\psi_{0}\right\rangle=0
$$

The Poisson bracket of $\Theta$ and $\bar{\Theta}$ is

$$
i\{\Theta, \bar{\Theta}\}=\phi \bar{\phi}+c \bar{c}-i c \bar{c}(\phi \bar{\gamma}-\gamma \bar{\phi})+\frac{3}{4} \gamma \bar{\gamma} c^{2} \bar{c}^{2}
$$

When $\gamma=0$, this is simply the $O S p(1,1 \mid 2)$-invariant form. We can prove the relation similar to (9),

$$
\lim _{\beta \rightarrow \infty} \exp (-\beta i\{\Theta, \bar{\Theta}\})=-\pi \delta(\phi) \delta(\bar{\phi}) \delta(c) \delta(\bar{c})
$$

To prove (5), we integrate the left side against a test function $\varphi(c, \bar{c})$, scale the ghosts and take the limit outside the integral,

$$
\begin{aligned}
& \lim _{\beta \rightarrow \infty} \int d c d \bar{c} \varphi(c, \bar{c}) \exp (-\beta i\{\Theta, \bar{\Theta}\}) \\
=\lim _{\beta \rightarrow \infty} \int \frac{d c^{\prime} d \bar{c}^{\prime}}{\beta} & \varphi\left(\frac{c^{\prime}}{\sqrt{\beta}}, \frac{\overline{c^{\prime}}}{\sqrt{\beta}}\right) e^{-c^{\prime} \bar{c}^{\prime}}(1-\beta \phi \bar{\phi}) \\
& \times\left(1+i c^{\prime} \bar{c}^{\prime}(\phi \bar{\gamma}-\gamma \bar{\phi})-\frac{3}{4 \beta}\left(c^{\prime} \bar{c}^{\prime}\right)^{2} \gamma \bar{\gamma}-\left(c^{\prime} \bar{c}^{\prime}\right)^{2} \phi \bar{\gamma} \gamma \bar{\phi}\right) \\
= & -\pi \phi \bar{\phi} \varphi(0,0) .
\end{aligned}
$$

We prove the general case of (9) in the next section.

\section{The General Case}

We now consider a general constrained system with $N$ fermionic and $M$ bosonic constraints satisfying the assumption of the method. The general form of the BRST charge $\Theta$ is

$$
\begin{aligned}
& \Theta=c_{I} \phi_{I}+\xi_{i} a_{i}+\sum_{\substack{n=0 \\
n+m \geq 1}}^{\infty} \sum_{\substack{m=0 \\
n}}^{M} \bar{c}_{\bar{I}_{1}} \cdots \bar{c}_{\bar{I}_{n}} c_{I_{1}} \cdots c_{I_{n}} \bar{\xi}_{\bar{\iota}_{1}} \cdots \bar{\xi}_{\bar{\iota}_{m}} \xi_{i_{1}} \cdots \xi_{i_{m}} \\
& \times\left(c_{I_{n+1}} \Xi^{\bar{I}_{1} \cdots \bar{I}_{n} I_{1} \cdots I_{n+1} \bar{\imath}_{1} \cdots \bar{\imath}_{m} i_{1} \cdots i_{m}}+\xi_{i_{m+1}} X^{\bar{I}_{1} \cdots \bar{I}_{n} I_{1} \cdots I_{n} \bar{\imath}_{1} \cdots \bar{z}_{m} i_{1} \cdots i_{m+1}}\right),
\end{aligned}
$$

where the indices run $I=1, \ldots, N$ and $i=1, \ldots, M$. We write this schematically as

$$
\Theta=c \phi+\xi a+\sum_{n, m} \bar{c}^{n} c^{n+1} \bar{\xi}^{m} \xi^{m} \Xi_{n, m}+\sum_{n, m}{ }^{\prime} \bar{c}^{n} c^{n} \bar{\xi}^{m} \xi^{m+1} X_{n, m},
$$

where $c^{n}$, for instance, denotes $c_{I_{1}} c_{I_{2}} \cdots c_{I_{n}}$ and the primed sum $\sum_{n, m}^{\prime}$ is a sum on all multi-indices of 
positive length, $\{n+m \geq 1, n \geq 0, M \geq m \geq 0\}$. From (1) it follows that the general BRST Laplacian is

$$
\begin{aligned}
i\{\Theta, \bar{\Theta}\}= & a_{i} \bar{a}_{\bar{\imath}}+\phi_{I} \bar{\phi}_{\bar{I}}+i \xi_{i}\left\{a_{i}, \bar{a}_{\bar{\jmath}}\right\} \bar{\xi}_{\bar{\jmath}}+i c_{I}\left\{\phi_{I}, \bar{\phi}_{\bar{J}}\right\} \bar{c}_{\bar{J}}+i \xi_{i}\left\{a_{i}, \bar{\phi}_{\bar{J}}\right\} \bar{c}_{\bar{J}}+i c_{I}\left\{\phi_{I}, \bar{a}_{\bar{\jmath}}\right\} \bar{\xi}_{\bar{\jmath}} \\
& + \text { terms quadratic in ghosts, times constraints } \\
& + \text { terms with more than two ghosts, }
\end{aligned}
$$

which we rewrite using DeWitt's supermatrix notation, ${ }^{[17]}$

$$
i\{\Theta, \bar{\Theta}\}=a \bar{a}+\phi \bar{\phi}+i \xi \mathrm{A} \bar{\xi}+i c \mathrm{~B} \bar{c}+i \xi \mathrm{C} \bar{c}+i c \mathrm{D} \bar{\xi}+\tilde{\Delta} .
$$

The last term, $\tilde{\Delta}$, contains all of the higher-order pieces.

To make the calculation of $e^{-\beta i\{\Theta, \bar{\Theta}\}}$ in the general case similar to the case of a single fermionic constraint considered above, it is useful to rescale the bosonic and fermionic constraints and ghosts,

$$
\begin{aligned}
& a=a^{\prime} / \sqrt{\beta}, \quad \bar{a}=\bar{a}^{\prime} / \sqrt{\beta}, \quad c=c^{\prime} / \sqrt{\beta}, \quad \bar{c}=\bar{c}^{\prime} / \sqrt{\beta} \\
& \phi=\phi^{\prime} / \sqrt{\beta}, \quad \bar{\phi}=\bar{\phi}^{\prime} / \sqrt{\beta}, \quad \xi=\xi^{\prime} / \sqrt{\beta}, \quad \bar{\xi}=\bar{\xi}^{\prime} / \sqrt{\beta} .
\end{aligned}
$$

We find

$$
e^{-\beta i\{\Theta, \bar{\Theta}\}}=\exp \left(-\beta \phi \bar{\phi}-\beta a \bar{a}-i \xi^{\prime} \mathrm{A} \bar{\xi}^{\prime}-i c^{\prime} \mathrm{B} \bar{c}^{\prime}-i \xi^{\prime} \mathrm{C} \bar{c}^{\prime}-i c^{\prime} \mathrm{D} \bar{\xi}^{\prime}-\beta \tilde{\Delta}\right)
$$

which we rewrite in the suggestive form

$$
e^{-\beta i\{\Theta, \bar{\Theta}\}}=\exp \left(-\beta \phi \bar{\phi}-\beta a \bar{a}-i \xi^{\prime}\left(\mathrm{A}-\mathrm{CB}^{-1} \mathrm{D}\right) \bar{\xi}^{\prime}-i\left(c^{\prime}+\xi^{\prime} \mathrm{CB}^{-1}\right) \mathrm{B}\left(\bar{c}^{\prime}+\mathrm{B}^{-1} \mathrm{D} \bar{\xi}^{\prime}\right)-\beta \tilde{\Delta}\right)
$$

The last piece, $\beta \tilde{\Delta}$, is the sum of the terms, $\beta \tilde{\Delta}=\beta \tilde{\Delta}^{(1)}+\beta \tilde{\Delta}^{(2)}$,

$$
\begin{aligned}
\beta \tilde{\Delta}^{(1)}=\sum_{n, m}^{\prime} & \left(a^{\prime} \bar{c}^{\prime n+1} c^{\prime n} \bar{\xi}^{\prime m-1} \xi^{\prime m} \bar{\Xi}_{n, m} \beta^{-n-m+\frac{1}{2}}\right. \\
& +i \bar{c}^{\prime n+1} c^{\prime n} \bar{\xi}^{\prime m} \xi^{\prime m+1}\left\{a, \bar{\Xi}_{n, m}\right\} \beta^{-n-m} \\
& +a^{\prime} \bar{c}^{\prime n} c^{\prime n} \bar{\xi}^{\prime m} \xi^{\prime m} \bar{X}_{n, m} \beta^{-n-m+\frac{1}{2}} \\
& +i \bar{c}^{\prime n} c^{\prime n} \bar{\xi}^{\prime m+1} \xi^{\prime m+1}\left\{a, \bar{X}_{n, m}\right\} \beta^{-n-m} \\
& +\phi^{\prime} \bar{c}^{\prime n} c^{\prime n} \bar{\xi}^{\prime m} \xi^{\prime m} \bar{\Xi}_{n, m} \beta^{-n-m+\frac{1}{2}} \\
& +i \bar{c}^{\prime n+1} c^{\prime n+1} \bar{\xi}^{\prime m} \xi^{\prime m}\left\{\phi, \bar{\Xi}_{n, m}\right\} \beta^{-n-m} \\
& +\phi^{\prime} \bar{c}^{\prime n-1} c^{\prime n} \bar{\xi}^{\prime m+1} \xi^{\prime m} \bar{X}_{n, m} \beta^{-n-m+\frac{1}{2}} \\
& \left.+i \bar{c}^{\prime n} c^{\prime n+1} \bar{\xi}^{\prime m+1} \xi^{\prime m}\left\{\phi, \bar{X}_{n, m}\right\} \beta^{-n-m}\right) \\
& +c . c .
\end{aligned}
$$




$$
\begin{aligned}
\beta \tilde{\Delta}^{(2)}=\sum_{n, m}^{\prime} \sum_{k, \ell}^{\prime} & \left(\bar{c}^{\prime n+k} c^{\prime n+k} \bar{\xi}^{\ell+m} \xi^{\ell+m} \Xi_{n, m} \Xi_{k, \ell} \beta^{1-k-n-\ell-m}\right. \\
& +\bar{c}^{\prime n+k+1} c^{\prime n+k+1} \bar{\xi}^{\prime \ell+m-1} \xi^{\prime \ell+m-1} \Xi_{n, m} \bar{\Xi}_{k, \ell} \beta^{1-k-n-\ell-m} \\
& +i \bar{c}^{\prime n+k+1} c^{\prime n+k+1} \bar{\xi}^{\prime \ell+m} \xi^{\prime \ell+m}\left\{\Xi_{n, m}, \bar{\Xi}_{k, \ell}\right\} \beta^{-k-n-\ell-m} \\
& +\bar{c}^{\prime n+k-1} c^{\prime n+k} \bar{\xi}^{\ell+m+1} \xi^{\prime \ell+m} \Xi_{n, m} \bar{X}_{k, \ell} \beta^{1-k-n-\ell-m}+c . c . \\
& +\bar{c}^{\prime n+k} c^{\prime n+k+1} \bar{\xi}^{\ell \ell+m} \xi^{\prime \ell+m-1} \Xi_{n, m} \bar{X}_{k, \ell} \beta^{1-k-n-\ell-m}+c . c . \\
& +i \bar{c}^{\prime n+k} c^{\prime n+k+1} \bar{\xi}^{\prime \ell+m+1} \xi^{\prime \ell+m}\left\{\Xi_{n, m}, \bar{X}_{k, \ell}\right\} \beta^{-k-n-\ell-m}+c . c . \\
& +\bar{c}^{\prime n+k-1} c^{\prime n+k-1} \bar{\xi}^{\prime \ell+m+1} \xi^{\prime \ell+m+1} X_{n, m} \bar{X}_{k, \ell} \beta^{1-n-k-\ell-m} \\
& +\bar{c}^{\prime n+k} c^{\prime n+k} \bar{\xi}^{\prime \ell+m} \xi^{\ell+m} X_{n, m} \bar{X}_{k, \ell} \beta^{1-n-k-\ell-m} \\
& \left.+i \bar{c}^{\prime n+k} c^{\prime n+k} \bar{\xi}^{\ell \ell+m+1} \xi^{\prime \ell+m+1}\left\{X_{n, m}, \bar{X}_{k, \ell}\right\} \beta^{-n-k-\ell-m}\right)
\end{aligned}
$$

The terms in (8) and (9) above are all at least $\mathcal{O}\left(\beta^{-\frac{1}{2}}\right)$, which means that they can be ignored in the limit $\beta \rightarrow \infty$. To obtain the delta function relation analogous to the Senjanovic measure (9), we integrate against a test function $\varphi$ of the bosonic variables $c, \bar{c}, a, \bar{a}$. The integral we wish to evaluate is

$$
\begin{aligned}
I_{\beta}= & \int d^{N} c d^{N} \bar{c} d^{M} a d^{M} \bar{a} e^{-\beta i\{\Theta, \bar{\Theta}\}} \varphi(c, \bar{c}, a, \bar{a}) \\
= & \int \frac{d^{N} c^{\prime} d^{N} \bar{c}^{\prime} d^{M} a^{\prime} d^{M} \bar{a}^{\prime}}{\beta^{N+M}} \varphi\left(\frac{c^{\prime}}{\sqrt{\beta}}, \frac{\bar{c}^{\prime}}{\sqrt{\beta}}, \frac{a^{\prime}}{\sqrt{\beta}}, \frac{\bar{a}^{\prime}}{\sqrt{\beta}}\right) \\
& \left.\left.\times e^{-\left(\beta \phi \bar{\phi}+a^{\prime} \bar{a}^{\prime}+i \xi^{\prime}\left(\mathrm{A}-\mathrm{CB}^{-1} \mathrm{D}\right) \bar{\xi}^{\prime}+i\left(c^{\prime}+\xi^{\prime} \mathrm{CB}-1\right.\right.}\right) \mathrm{B}\left(\bar{c}^{\prime}+\mathrm{B}^{-1} \mathrm{D} \bar{\xi}^{\prime}\right)+\mathcal{O}\left(\beta^{-\frac{1}{2}}\right)\right)
\end{aligned}
$$

which, upon a shift of the ghost variables, $c^{\prime} \rightarrow c^{\prime}-\xi^{\prime} \mathrm{CB}^{-1}$, becomes

$$
\begin{aligned}
I_{\beta}=(-1)^{N+M} \int & \frac{d^{N} c^{\prime} d^{N} \bar{c}^{\prime} d^{M} a^{\prime} d^{M} \bar{a}^{\prime}}{\beta^{N+M}} \varphi\left(\frac{c^{\prime}}{\sqrt{\beta}}, \frac{\bar{c}^{\prime}}{\sqrt{\beta}}, \frac{a^{\prime}}{\sqrt{\beta}}, \frac{\bar{a}^{\prime}}{\sqrt{\beta}}\right) \beta^{N} \delta^{N}(\phi) \delta^{N}(\bar{\phi}) \\
& \times \beta^{M} \delta^{M}(\xi) \delta^{M}(\bar{\xi}) \operatorname{det}\left[i\left(\mathrm{~A}-\mathrm{CB}^{-1} \mathrm{D}\right)\right] e^{-a^{\prime} \bar{a}^{\prime}} e^{-i c^{\prime} \mathrm{B}^{\prime}}+\mathcal{O}\left(\beta^{-\frac{1}{2}}\right) .
\end{aligned}
$$

Because $\varphi$ is a test function, we obtain in the limit

$$
\lim _{\beta \rightarrow \infty} I_{\beta}=(-\pi)^{N+M} \delta^{N}(\phi) \delta^{N}(\bar{\phi}) \delta^{M}(\xi) \delta^{M}(\bar{\xi}) \operatorname{det}\left[i\left(\mathrm{~A}-\mathrm{CB}^{-1} \mathrm{D}\right)\right](\operatorname{det}(i \mathrm{~B}))^{-1} \varphi(\mathbf{0}, \mathbf{0}, \mathbf{0}, \mathbf{0}),
$$

which proves the general case of (9),

$$
\begin{aligned}
\lim _{\beta \rightarrow \infty} e^{-\beta i\{\Theta, \bar{\Theta}\}}= & (-\pi)^{N+M} \delta^{N}\left(\phi_{I}\right) \delta^{N}\left(\bar{\phi}_{\bar{J}}\right) \delta^{M}\left(\xi_{i}\right) \delta^{M}\left(\bar{\xi}_{\bar{\jmath}}\right) \operatorname{sdet}\left(\begin{array}{cc}
i\left\{a_{i}, \bar{a}_{\bar{\jmath}}\right\} & i\left\{a_{i}, \bar{\phi}_{\bar{J}}\right\} \\
i\left\{\phi_{I}, \bar{a}_{\bar{\jmath}}\right\} & i\left\{\phi_{I}, \bar{\phi}_{\bar{J}}\right\}
\end{array}\right) \\
& \times \delta^{N}\left(\operatorname{Re} c_{I}\right) \delta^{N}\left(\operatorname{Im} \bar{c}_{\bar{J}}\right) \delta^{M}\left(\operatorname{Re} a_{i}\right) \delta^{M}\left(\operatorname{Im} \bar{a}_{\bar{\jmath}}\right) .
\end{aligned}
$$

In summary, our main result is that the harmonic BRST-BFV method introduced in ref. [1] generalizes to the case of mixed bosonic and fermionic constraints. There is no problem in extending the operator 
formalism, but it is not trivial to show that the extension also works for the path integral. It is possible that the relation (13) is only valid for test functions and not for rapidly decreasing functions as well. This is because the bosonic part of the exponent $-\beta i\{\Theta, \bar{\Theta}\}$ might not be negative definite, although the quadratic piece by itself is negative definite. For test functions, this is irrelevant because in the limit the quadratic piece dominates all others on any finite interval. In the case of purely bosonic constraints, this is not an issue because the higher order pieces are fermionic and have no convergence problem. To be rigorous about a particular path integral, one must check in that specific case that the result also holds for functions of rapid decrease.

Our modification of the path integral and our limit argument are very similar to the method of equivariant localization recently introduced by Dykstra, Lykken and Raiten. ${ }^{[18]}$ Invariance under the change of variables generated by the holomorphic BRST charge $\Theta$ in our formalism corresponds closely to the invariance under the equivariant exterior derivative $d_{\chi}$ in the formalism of ref. [18].

The extension of the formalism to the reducible case is an open problem, but one whose solution is quite likely to follow the standard reducible BRST quantization for real constraints.

\section{ACKNOWLEDGEMENTS}

It is a pleasure to thank Bernice Durand for her careful reading of this paper and her many useful suggestions. This work was supported in part by DOE grant No. DE-AC02-76-ER00881.

\section{REFERENCES}

1. T.J. Allen, Phys. Rev. D43 (1991) 3442.

2. W. Kalau, Int. J. Mod. Phys. A8 (1993) 391.

3. A. Ashtekar, Lectures on Non-perturbative Canonical Gravity, Lecture notes prepared in collaboration with R.S. Tate. (World Scientific Books, Singapore, 1991);

C. Rovelli, Class. Quantum Grav. 8 (1991) 1613.

4. E. Nissimov, S. Pacheva, and S. Solomon, Nucl. Phys. B297 (1988) 349.

5. T.J. Allen, Caltech Ph.D. Thesis (1988).

6. S. Aoyama, J. Kowalski-Glikman, J. Lukierski and J.W. van Holten, Phys. Lett. 216B (1989) 133. 
7. A.P. Balachandran, S. Borchardt and A. Stern, Phys. Rev. D17 (1978) 3247;

A.P. Balachandran, G. Marmo, B.-S. Skagerstam and A. Stern, Gauge Symmetries and Fibre Bundles, Springer-Verlag Lecture Notes in Physics 188, (Springer-Verlag, Berlin and Heidelberg, 1983);

A.P. Balachandran, M. Bourdeau, and S. Jo, Int. J. Mod. Phys. A5 (1990) 2423;

E. Gates, R. Potting, C. Taylor, and B. Velikson, Phys. Rev. Lett. 63 (1989) 2617.

8. J. Kowalski-Glikman, Phys. Lett. B245 (1990) 79. "On Gupta-Bleuler Quantization of Hamiltonian Systems with Anomalies," hep-th preprint 9211028, to appear in Ann. Phys.

9. Z. Hasiewicz, J. Kowalski-Glikman, J. Lukierski, and J.W. van Holten, Phys. Lett. 217B (1989) 95.

10. R. Marnelius, Nucl. Phys. B 370 (1992) 165.

11. F. A. Berezin and M. S. Marinov, Ann. Phys. (N.Y.) 104 (1977) 336;

F. Bordi and R. Casalbuoni, Phys. Lett. B 93 (1980) 308;

T. J. Allen, Phys. Lett. B 214 (1988) 87.

12. P. Senjanovic, Ann. Phys. (N.Y.) 100 (1976) 227.

13. M. Henneaux, Phys. Rep. 126 (1985) 1.

14. M. Henneaux, Classical Foundations of BRST Symmetry, (Bibliopolis, Naples, 1988) pp. 45-50.

15. Jan Govaerts, Hamiltonian Quantization and Constrained Dynamics, (Leuven University Press, Leuven, 1991) p. 69.

16. E. S. Fradkin and G. A. Vilkovisky, Phys. Lett. 55B (1975) 224;

I. A. Batalin and G. A. Vilkovisky, Phys. Lett. 69B (1977) 309;

E. S. Fradkin and T. E. Fradkina, Phys. Lett. 72B (1978) 343.

17. Bryce DeWitt, Supermanifolds, (Cambridge University Press, Cambridge, 1992) p.20.

18. H.M. Dykstra, J.D. Lykken and E.J. Raiten, "Exact Path Integrals by Equivariant Localization," FERMI-PUB-92/383-T, UMHEP-384, hep-th preprint 9212126. 